\title{
La influencia social 2.0 en los programas magazín de las radios generalistas españolas con relación a su audiencia
}

\author{
Joan Francesc FondEVILA GASCÓN \\ Universitat Pompeu Fabra (UPF) \\ joanfrancesc.fondevila@upf.edu \\ Carles LAmelo VARELA \\ Universitat Abat Oliba-CEU \\ clamelov@uao.es
}

Recibido: 9 de abril de 2014

Aceptado: 14 de octubre de 2014

\section{Resumen}

La radio es un medio de comunicación que tradicionalmente ha resistido el embate de los nuevos medios. Lo mismo está ocurriendo con Internet, con el añadido de la diversidad de interacciones entre ambos. En este artículo analizamos las variables de audiencia y el índice Klout, considerado oficiosamente como el sintetizador de la influencia de un individuo o una entidad en redes sociales. Incluimos los números absolutos y los porcentajes de la cantidad de seguidores en Twitter de cada cadena de radio generalista, el índice de influencia y la audiencia según el EGM. El análisis comparado de esos datos pone en duda la relación entre seguidores, oyentes y el indicador Klout, ya que las correlaciones detectadas, pese a tender a elevadas, no alcanzan ese nivel.

Palabras clave: Radio, redes sociales, audiencia, Internet, medios sociales.

\subsection{Social influence of the Spanish talk radio programs in relation to its audience}

\begin{abstract}
Radio is a medium that has traditionally resisted the onslaught of new media. The same is happening with the Internet, with the addition of the diversity of interactions between them. In this paper we analyze the variables of audience and Klout index, informally considered as the synthesizer of the influence of an individual or an entity in social networks. We include the absolute numbers and percentages of the amount of Twitter followers of each chain generalist radio, the influence index and the audience as the EGM. The comparative analysis of these data calls into question the relationship between followers and Klout listeners' indicator, since the detected correlations; although high tend to not reach that level.
\end{abstract}

Keywords: Radio, social networks, audience, Internet, social media.

\section{Referencia normalizada}

FONDEVILA GASCÓN, Joan Francesc y LAMELO VARELA, Carles (2015): "La influencia social 2.0 en los programas magazín de las radios generalistas españolas con relación a su audiencia". Estudios sobre el Mensaje Periodístico. Vol. 21, Núm. 2 (julio-diciembre), págs.: 813-831. Madrid, Servicio de Publicaciones de la Universidad Complutense.

Sumario: 1. Las redes sociales y la generación de contenidos radiofónicos. 2. Los seguidores de Twitter en la radio española. 3. La importancia de la métrica de la influencia digital. 4. Trabajo de campo: Las influencia digital Klout y la audiencia de los magazines de las radios españolas. 5. Conclusiones. 6. Referencias bibliográficas. 


\section{Las redes sociales y la generación de contenidos radiofónicos}

En los últimos años, la mayoría de programas magazín de la radio española han incorporado las redes sociales a sus contenidos. Facebook (Holahan 2008)y principalmente Twitter (Ju, Jeong y Chyi, 2013) se han revelado como nuevos canales de comunicación (Olmstead, Mitchell y Rosenstiel, 2011) con los oyentes porque ayudan a crear engagement (Alexander, 2013) con la audiencia y sirven como herramienta indispensable para difundir información a tiempo real como servicio adicional a la programación radiofónica convencional (Martínez Costa, Moreno y Amoedo, 2012) y como agregador de usuarios y generador de tráfico hacia las páginas web de las cadenas (Herrera Damas y Requejo, 2012). En este proceso, los oyentes juegan un papel capital puesto que discriminan a través del timeline de Twitter las fuentes de información que les generan confianza y credibilidad (O' Conor, 2012) mientras comparten, comentan y aportan datos (Hermida 2010) en una comunicación que es transmedia (Scolari, 2009), dentro de un entorno multimedia (Fondevila Gascón, 2010) y multipantalla (Rodríguez Fidalgo y Sánchez Mera, 2013) lo que convierte a los usuarios en prosumers (Meerman Scott, 2010). Al igual que la radio, Twitter "facilita intercambio de información en tiempo real" (Hermida, 2010) al tiempo que "permite que un gran número de usuarios se comuniquen entre sí de forma simultánea y en tiempo real, a partir de una relación asimétrica entre amigos y seguidores".

Lejos de entrar en rivalidades, las redes sociales y la radio se complementan y se retroalimentan. Gracias a Twitter las emisoras pueden hacer promoción de sus programas y fidelizar a los oyentes a través de la gestión de comunidades o del hipervínculo hacia servicios de podcasting (Sellas, Radio y redes sociales: los magazines matinales en Twitter 2013) que define este servicio asegurando que "Twitter es un servicio de microblogging que permite a sus usuarios leer y enviar textos pequeños de una longitud máxima de 140 caracteres denominados 'tuits"'. Se ha convertido en pocos años -desde 2006- en elemento esencial para la comunicación digital y ha impactado de manera decidida en el ejercicio del periodismo (Campos Freire, 2008). Desde su aparición, la plataforma de Jack Dorsey se ha posicionado como espacio de información "newsful" (Chyi y Chadha, 2012) y como fuente de documentación (Traver Vallés y Cobarsí Morales 2012) mientras que Facebook ha ido perdiendo posiciones en este flanco para centrarse en su vertiente social y de comunidad (Requejo y Herrera, 2011).

La red de microblogging (Java 2007) triunfa gracias a la sencillez de su interfaz (Abejón Mendoza, Sastre Asensio y Linares Rodríguez, 2012) y facilita de manera decidida la creación de tráfico de usuarios únicos hacia la edición web (Gleason, 2010) del medio de comunicación (Jeong, 2013) mientras ayuda a que la interacción entre los oyentes y los programas de radio que escuchan sea más directa permitiendo incluso que escuchantes alejados geográficamente interactúen entre ellos durante la emisión de un programa o después de su finalización. La vida de los contenidos radiofónicos se alarga en el podcasting y también en las redes sociales donde se promueve el consumo a la carta de tertulias, entrevistas, secciones y programas; especialmente aquellos cuyos contenidos son más especializados o van menos ligados a la actualidad inmediata. En éstos, las redes sociales juegan un papel trascendental en la difusión de noticias a tiempo real y también en la participación de la audiencia que vuelca sus 
opiniones o aporta datos ejerciendo la función de prosumidor o prosumer, neologismo que surge al formar un acrónimo por la fusión original de las palabras inglesas producer (productor) y consumer (consumidor) (P. Herrera 2008).

La dinámica voraz (Naaman, Becker y Gravano, 2011) de las sociedades 2.0 exige a los generadores de contenidos (Picard, 2006) que ofrezcan información (Gutiérrez Atala y Arriagada, 2012) de manera constante (Harbison, 2010) y actualizada (Holahan, 2008) y que estén en contacto con los oyentes estableciendo espacios de diálogo que ayuden a fomentar y crear comunidad manteniendo una "escucha activa" (Ferreras Rodríguez, 2011). Los medios deben evitar convertir los mensajes que difunden en redes y plataformas sociales en una versión digital de sus contenidos (Parr, 2009) habituales. Según el estudio de Herrera y Requejo (Herrera Damas y Requejo, 2012), el $67 \%$ de las actualizaciones de perfiles de Twitter de las emisoras de radio generalistas se dedicaron a difundir información mientras que un 26,6\% tuvo una intencionalidad promocional. Se demuestra imprescindible plantear una estrategia que esté fundamentada en los códigos de la llamada netiqueta (Torres Vilatarsana, 2004) y que el uso que se haga de las redes sociales para transmitir información, estimular la participación o promocionar (Armstrong y Gao, 2010) los contenidos (Sellas, El Podcasting: la (r) evolución sonora 2011) responda a la lógica del social media, cuyo objetivo es, por encima de otras consideraciones, la creación de comunidades 2.0: "hay que aportar valor para obtener atención a cambio, y hay que hacerlo de modo regular para conseguir una comunidad valiosa" (Orihuela, 2011). Este camino se aventura más fácil para la radio, un soporte con una amplia presencia en el mapa mediático español y que goza de altas tasas de popularidad y credibilidad entre la ciudadanía. Según el Estudio General de Medios (AIMC 2013), la radio tiene una penetración del $61,7 \%$ de la población española mientras que el Baròmetre (Centre d'Estudis d'Opinió 2012) del CEO de la Generalitat de Cataluña señala que el 44,5\% de los ciudadanos catalanes se informa de los temas políticos a través de la radio.

Las redes sociales son ya un elemento esencial para la transmisión de noticias de última hora (Holahan 2008), tarea que hasta la aparición de los medios digitales realizaba con celeridad la radio a través de sus boletines informativos (Fraticelli, 2011) y los flashes que interrumpen la programación ante acontecimientos noticiables de gran relevancia. Tanto en la escucha radiofónica como en su proyección 2.0 a través de las social network, el oyente es quien elige a quién escucha a través del transistor y también a quién sigue en Twitter: "Cuando los usuarios eligen darle a 'me gusta'o seguir a un periódico en las redes sociales, están de acuerdo en recibir su alimentación de noticias" (Ju, Jeong y Chyi, 2013).

La expansión del fenómeno Twitter o su contracción dependerá de cómo evolucione la red semántica 3.0 (P. Castells 2005); la evolución de la web social (Celaya, 2008) que traspasa esta dimensión para fundamentarse en las bases de datos que intuyen lo que busca el usuario apoyándose en la inteligencia artificial.

\section{Los seguidores de Twitter en la radio española}

Muchos internautas, y por extensión también los medios de comunicación, tienen una cierta obsesión por incrementar el número de seguidores, amigos, círculos o fans en 
los perfiles de los programas y cadenas, aunque la media de contactos de los usuarios convencionales en las redes sociales está en 195 (Insites Consulting, 2011). El volumen de seguidores no es tan importante como la creación de comunidad y la interactividad que se establece con los oyentes. Ahí es donde la métrica de la influencia en redes sociales se revela útil para la monitorización del éxito 2.0 de los contenidos radiofónicos. Para televisión empiezan a aparecer en Estados Unidos -y ahora también en España- empresas especializadas en el cómputo de la viralidad televisiva tales como Tuitele ${ }^{1}$. Otros operadores tradicionales como Nielsen ofrecen servicios similares en diferentes países ${ }^{2}$ atendiendo a la necesidad de las marcas de conocer el impacto que tienen sus spots televisivos entre un público socialmente conectado; sobre todo porque la participación Facebook y Twitter comporta un componente psicológicamente diagnosticado (Watts, 2004) y es esencial para las estrategias de inbound marketing (Zarrella, 2010) y para la generación de engagement.

En octubre de 2013, Twitter declaraba tener 218 millones de usuarios activos al mes en todo el mundo (Pozzi, 2012) y se estima que en España supera los 4,5 millones. Existe una significativa diferencia entre Facebook y Twitter. La red de microblogging continúa en expansión y creció un $40 \%$ en la segunda parte del año 2012, mientras que Facebook subió un 33\%. En España, el 30\% de los usuarios de Internet utilizan Twitter mientras que en Estados Unidos son el 20\%. Según el estudio ComsCore de abril de 2013 (ComScore 2013), los internautas norteamericanos dedican 42,8 horas al mes conectados a Internet; en Europa, esa cifra se reduce a 26,9 horas, por encima de la media mundial fijada en 24,7 horas. Los españoles son el $4 \%$ de los internautas europeos, con más de 22 millones de usuarios y están conectados a la Red una media de 23,8 horas al mes.

En diciembre de 2012, Facebook tenía en España 17 millones de usuarios únicos que se conectaban a través de ordenador y Twitter alcanzaba la cifra de 5,6 millones, a los que hay que añadir los que se conectan en movilidad, que en el caso de la red social ideada por Mark Zuckerberg son 13,8 millones; el triple que Twitter, que conseguía 4,5 millones: una cifra que se dobló en su cómputo interanual. Estos datos muestran la dimensión estratégica que tiene para las marcas la comunicación en social media y su relevancia para los movimientos de activismo político (González Mendoza y Petersen, 2010). Las emisoras de radio, en tanto que marcas, y sus clientes -los anunciantes- necesitan un baremo que les ayude a aproximarse lo más posible a un análisis empírico de su influencia en redes sociales, más allá del número de usuarios que pueden ser inactivos y alejados del concepto de comunidad que recomiendan las estrategias de social media. Se da la paradoja que algunos medios de comunicación internacionales como en New York Times tienen muchos más seguidores en Twit-

1 Cuenta con servicios de pago, aunque puede consultarse gratuitamente la lista de programas más seguidos en redes sociales en línea a través de la página web http://www.tuitele.tv/audiencia-social/ (Consulta: noviembre, 2013).

2 Aunque se trata de un servicio incipiente, el portal web de esta multinacional de los estudios de mercado explica el funcionamiento de su nuevo panel de monitorización de social media en línea: http://www.nielsen.com/us/en/press-room/2013/nielsen-launches-nielsen-twittertv-ratings.html (Consulta: noviembre, 2013). 
ter que lectores porque miles de usuarios de Internet deciden seguirle como estrategia de marca personal para dotarse de una pátina de credibilidad en su proyección social online y offline, aunque en realidad no lean asiduamente el diario.

\section{La importancia de la métrica de la influencia digital}

El exceso de información en Internet lleva a la desinformación a través de la saturación del usuario. Para poder cribar los contenidos se perfila la figura del curador que viene a reforzar el papel del documentalista y del periodista que se pone al servicio de los generadores y difusores de contenidos y los influencers (Gillin, 2009). Los medios de comunicación convencionales siguen siendo quienes marcan la agenda (Cobb y Elder, 1972) y la construyen a través del relato informativo y la contextualización de los hechos noticiosos, también en las redes sociales. Como los perfiles en Facebook y Twitter se han convertido en herramientas de difusión de noticias y captación de tráfico hacia los servicios online y offline, podemos catalogar a los perfiles de las emisoras de radio como influyentes en el social media, además de serlo en los soportes tradicionales. Javier Serrano-Puche (Serrano-Puche, 2012) describe este proceso asegurando que "los usuarios influyentes cumplen con la función bien de generar contenidos, bien de filtrarlos hacia otros usuarios sobre los que tienen cierta ascendencia".

Para medir la influencia de los perfiles de Twitter existen algunas metodologías como el Follower Ratio (TFF Ratio) $)^{3}$ o las combinaciones algorítmicas de servicios en línea como Klout, una herramienta de medición fundada en 2008 y que pretende convertirse en estándar, no sin cierta controversia (Livingston, 2010). Su funcionamiento es secreto pero se sabe que utiliza diversos parámetros para elaborar una monitorización diaria que puntúa a los perfiles y usuarios de redes sociales en una escala de 1 a 100 (Anger y Kitti, 2011), siendo el presidente norteamericano Barack Obama el paradigma de influencia 2.0 con un índice Klout de $99^{4}$, mientras que la puntuación media de los usuarios ronda los 20 puntos y el percentil 80 se sitúa por encima de 40 (Zhang, 2013).

A falta de un estándar académicamente consensuado, "Klout es actualmente la herramienta de medición de mayor aceptación social (alcanzó los 100 millones de usuarios conectados en septiembre de 2011) [...] basándose en más de 30 algoritmos que la empresa no ha dado a conocer". Serrano-Puche sitúa tres factores esenciales para la configuración del índice Klout de un usuario:

1) Alcance real: representa el número de personas sobre las que se ejerce influencia [...]. 2) Amplificación: se mide tomando en cuenta cómo los mensajes provocan conversación o son replicados: retuiteados, marcados como 'me gusta' [...]. 3) Im-

3 "TFF Ratio $=\mathrm{n}^{\circ}$ followers $/ \mathrm{n}^{\circ}$ following. Si el TFF Ratio: 1 ) es menor de 1: quiere decir que se esta tratando de conectar con usuarios, pero por el momento no se está consiguiendo, 2) es cercano a 1: se escucha y se es escuchado, 3) es igual o mayor que 2 : quiere decir que se es un líder en una comunidad, y que la gente quiere escuchar, 4) es mayor que 10: quiere decir que o se es un personaje conocido o un elitista al que le molesta el 'ruido' de Twitter". Cfr. (Elósegui 2011) citado en (Herrera y Requejo 2012).

4 Puede consultarse este dato en línea: http://klout.barackobama (Consulta: noviembre de 2013). 
pacto en la Red: determina la influencia que a su vez tienen los seguidores que están dentro del alcance real del usuario evaluado." (Elósegui, 2011)

Una vez establecidos los criterios que contribuyen a incrementar el índice Klout de un usuario, Serrano-Puche define los perfiles de los influenciadores: "el curador (muy participativo y propenso a compartir contenidos); el famoso (crea contenido, de tipo muy variado); el observador (fundamentalmente escucha y utiliza las redes sociales de manera ocasional) y el especialista (tiene una presencia online constante, difundiendo contenido específico)".

Los comunicadores que están al frente de los principales programas de radio de España son, a su vez, influenciadores a través de sus perfiles sociales. Aprincipios de noviembre de 2013, la periodista de Onda Cero Julia Otero ${ }^{5}$ tenía en Twitter 286.450 seguidores y un índice Klout de 78, a mucha distancia del perfil de su programa Julia en la Onda ${ }^{6}$ que aglutinaba a 57.568 seguidores con una influencia de 67, pese a ser el segundo programa vespertino más escuchado de la radio en España. La explicación se halla en el perfil mediático de Julia Otero y su condición de celebrity (Marwick, 2011) o "líder de opinión en audiencia social, aquel que en la emisión de un programa, consigue propagar más sus mensajes" (Congosto y Claes, 2013).

Existen otras herramientas en línea para monitorizar la influencia de los usuarios tales como PeerIndex, Kred o Traack, aunque el presente estudio considera a Klout como el servicio de referencia para medir la influencia en redes sociales de la "sociedad en red" descrita por Manuel Castells en 2001.

El presente artículo tiene como objetivo averiguar si existe una correlación entre la influencia social medida en el índice Klout de los principales programas magazín matinales y vespertinos y el número de oyentes que les consigna el Estudio General de Medios (EGM), principal monitorizador de las audiencias en la radio española. Para realizar una fotografía de esta influencia se recurrirá a métodos cuantitativos a partir de datos objetivos obtenidos del servidor de influencia Klout, contrastados con otras métricas de influencia y los datos que proporciona la tercera oleada del EGM del año 2013, que es la primera que se conoce en la temporada radiofónica 2013-2014. Se repetirá la métrica en las audiencias acumuladas de las principales cadenas de radio españolas: Cadena SER, Onda Cero, Radio 1 de RNE y Cope.

\section{Trabajo de campo: Las influencia digital Klout y la audiencia de los magazi- nes de las radios españolas}

La radio española mide su audiencia a través el Estudio General de Medios (EGM) (Nieto, 1994), un análisis estadístico cuantitativo que se realiza en tres oleadas anuales ${ }^{7}$ y se fundamenta en entrevistas y encuestas en un panel de hogares que se corresponde

5 Puede consultarse su perfil (@julia_otero) en línea htt://twitter.com/julia_otero (Consulta: 6 de noviembre de 2013).

6 Puede consultarse su perfil (@Juliaenlaonda) en línea: https://twitter.com/Julianelaonda (Consulta: 6 de noviembre de 2013).

7 Puede consultarse el calendario de estudio, resumen de resultados y procedimientos del estudio en la web de la Asociación para la Investigación de los Medios de comunicación (AIMC) disponible en línea en: www.aimc.es (Consulta: marzo de 2014). 
con el universo de oyentes de la radio en España. El trabajo de campo del EGM que se utiliza en este análisis se realizó entre el 4 de septiembre y el 12 de noviembre de 2013, según la Asociación para la Investigación de los Medios de Comunicación (AIMC) ${ }^{8}$ y se corresponde con la $3^{\mathrm{a}}$ ola del año, primera de la temporada radiofónica 2013-14.

La audiencia del medio radiofónico, de lunes a viernes, es de 24.874 .000 oyentes, un $2,3 \%$ menos que en la ola anterior y un $2,9 \%$ menos que en la misma ola del año 2012. En términos generales, la radio generalista consigue 11.661 .000 oyentes, un $4,3 \%$ menos que en la $2^{a}$ ola de 2013 , lo que constituye una bajada del $7,7 \%$ en comparación con el mismo período del año pasado.

La radio temática alcanza los 15.484 .000 oyentes en los días laborales, un dato prácticamente idéntico al registrado en la oleada anterior, tras un incremento tenue de 58.000 oyentes. Si segregamos los datos de la radio musical, ésta ha crecido en 39.000 nuevos oyentes hasta conseguir 14.425.000 oyentes. Si se comparan estos datos con las audiencias del año anterior, prácticamente no se han producido variaciones. La radio temática ha descendido un $1,2 \%$ y la temática musical un $0,2 \%$, datos poco significativos atendiendo al margen de error del EGM.

La ficha técnica del último año móvil disponible, correspondiente a Febrero-Noviembre de 2013, establece un universo de la población española de 14 o más años, analizada con la siguiente muestra:

Ficha Técnica del Estudio General de Medios (EGM) Año Móvil Feb-Nov $2013^{9}$

\begin{tabular}{|c|c|}
\hline Universo & Población de 14 o más años: 39.331 .000 individuos \\
\hline \multirow{5}{*}{ Muestra anual tres últimas olas } & 32.289 entrevistas multimedia \\
\hline & +46.931 monomedia radio \\
\hline & +42.677 monomedia prensa \\
\hline & +23.680 monomedia revistas \\
\hline & + 10.675 monomedia televisión) \\
\hline \multirow{3}{*}{ Método de recogida de información } & Entrevista "face to face" \\
\hline & $\begin{array}{l}\text { + entrevista telefónica para ampliación radio, prensa } \\
\text { y tv }\end{array}$ \\
\hline & $\begin{array}{l}\text { (+ entrevista "face to face"" para ampliación } \\
\text { revistas) }\end{array}$ \\
\hline Diseño muestral & $\begin{array}{l}\text { Selección aleatoria de hogares y elección de una } \\
\text { persona del hogar }\end{array}$ \\
\hline
\end{tabular}

La radio es el segundo medio con mayor penetración en España (AIMC 2013), tras la televisión. Su importancia en la configuración de la opinión pública es relevante, pese a que en la corriente del agenda setting y su evolución a través del agenda building se utiliza la prensa escrita como paradigma (McCombs, 2013). El medio ra-

8 Puede consultarse su metodología, calendario y resumen general en línea visitando www.aimc.es (Consulta: febrero de 2014) (AIMC 2013).

9 Fuente: AIMC, Asociación para la Investigación de los Medios de Comunicación [en línea] www.aimc.es (Consulta: diciembre de 2013). 
diofónico ejerce un papel dominante, especialmente en las capas altas de la sociedad. Según el EGM, la penetración en la clase alta alcanza el 72,5\%.

\begin{tabular}{|l|l|}
\hline $\begin{array}{l}\text { Penetración de la } \\
\text { radio en España }\end{array}$ & 24.180 .000 oyentes/día \\
\hline \multirow{2}{*}{ Por sexo } & Hombres: $66,2 \%$ \\
\hline \multirow{3}{*}{ Por edad } & Mujeres: $57,0 \%$ \\
\hline \multirow{4}{*}{ Por clase social } & $25-34$ años: $68,8 \%$ \\
\cline { 2 - 2 } & $35-44$ años: $69,9 \%$ \\
\cline { 2 - 2 } & $45-54$ años: $64,6 \%$ \\
\cline { 2 - 2 } & Alta: $72,5 \%$ \\
\cline { 2 - 2 } & Media-alta: $68,5 \%$ \\
\cline { 2 - 2 } & Media baja: $63,1 \%$ \\
\cline { 2 - 2 } & Baja: $53,0 \%$ \\
\hline
\end{tabular}

La radio, como los periódicos, compite con los nuevos medios digitales redibujando su papel (Baus Belau 2001) en este entorno cambiante.

Tabla 1. Penetración de los medios de comunicación en la audiencia española (\%). Fuente: Estudio General de Medios (EGM). Elaboración propia.

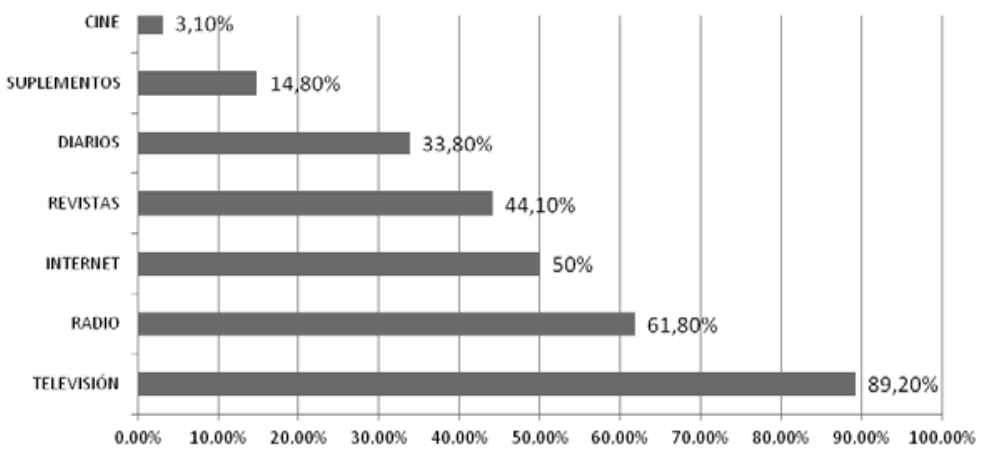

Desde el punto de vista publicitario, la radio es un medio creíble (González Conde, 2012) que genera enormes posibilidades narrativas (Comas, 2006) que se multiplican en la era digital gracias al podcasting (Martínez Costa, Moreno y Amoedo, 2012). En los últimos años, su penetración en la audiencia española (Tabla 1), lejos de aminorar, ha crecido pese a la irrupción de Internet (Cebrián Herreros 2008), que ha supuesto un auténtico desafío para la radio (Coyle, 2006). "La red incrementa, diversifica y amplía estas posibilidades tradicionales de participación de la audiencia de la radio, dándole mayor protagonismo y permitiendo también un diálogo o conversación entre los propios oyentes" (Martínez Costa, Moreno y Amoedo, 2012: 175).

${ }^{10}$ Fuente: AIMC, Asociación para la Investigación de los Medios de Comunicación [en línea] www.aimc.es (Consulta: diciembre de 2013) 
Tabla 2. Evolución de la penetración de la radio en España.

Fuente: Estudio General de Medios (EGM). Elaboración propia

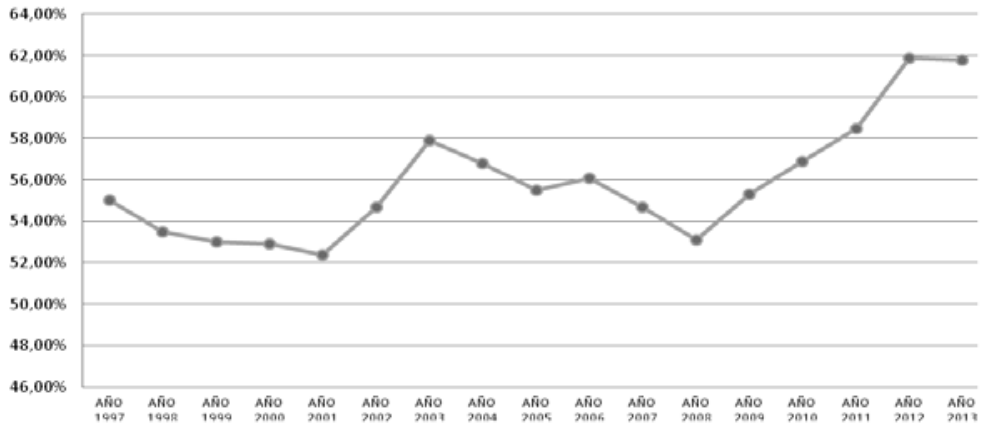

A lo largo de la serie histórica, la radio ha superado ampliamente el umbral del $50 \%$ de penetración en la audiencia (Tabla 2). Más de la mitad de los ciudadanos españoles mayores de trece años sintoniza cada día con alguna cadena, sea ésta especializada o generalista; esta tendencia que viene creciendo desde el año 2008 hasta sobrepasar el techo del año 2003, cuando la radio alcanzó una penetración del 58\%. A partir del año 2012 el crecimiento se modera o baja ligeramente, pero siempre por encima del $60 \%$, por lo que convendrá permanecer atentos a su evolución. Esa tendencia determinará si se han alcanzado cotas máximas, se contrae la demanda o bien continúa su expansión.

El Estudio General de Medios distingue dos grandes grupos de emisoras radiofónicas: las generalistas y las temáticas (Tabla 3). Las primeras combinan información y entretenimiento para atraer audiencias diversas (Franquet, 2008); las segundas van dirigidas a un segmento de la audiencia con contenidos monotemáticos informativos o musicales. Dentro de estas últimas, podemos situar las temáticas informativas como Radio 5-Todo Noticias o Catalunya Informació y aquellas cuyo contenido es puramente melódico como Los 40 Principales, Europa FM o Cadena Dial. Las radio-fórmulas musicales se basan en la repetición sistemática de una distribución categorizada de temas musicales designados por el llamado hot clock (Stephenson, Reese y Beadle, 2013), una estructura estable y repetitiva que también se aplica en las temáticas informativas. La radio generalista, por su parte, centra sus contenidos en información, entretenimiento, deportes, música y espacios de participación (Perona, 2009) a través de grandes programas magazín y espacios especializados (Gutiérrez y Huertas, 2003). La segmentación de contenidos y la división de operadores en estas tres categorías pone de manifiesto que su crecimiento es desigual, en conjunción con su éxito de audiencia. Según el Estudio General de Medios, la radio musical supera a la radio generalista en número de oyentes. 
Tabla 3. Número de oyentes de la radio generalista y temática.

Fuente: Estudio General de Medios (EGM). Elaboración propia.

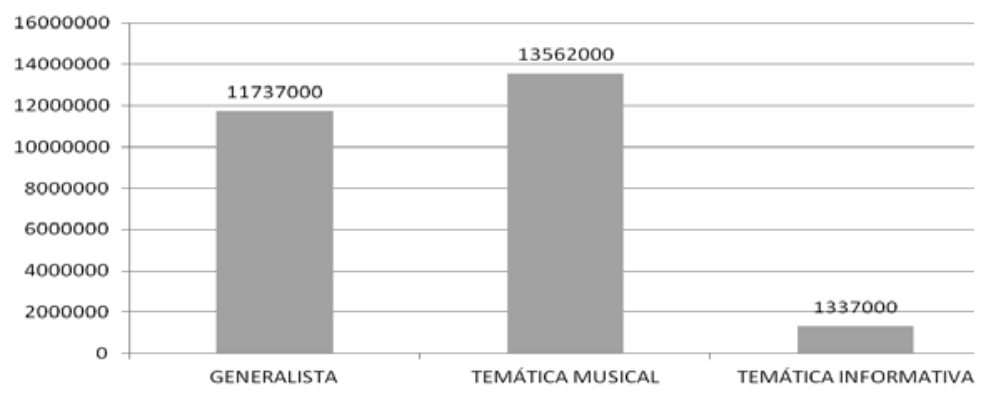

Un estudio de Videla Rodríguez y Piñeiro Otero (2013) demuestra que las cadenas de radio-fórmula musical aprovechan mejor el potencial de las redes sociales que las cadenas generalistas y las especializadas informativas. En términos de audiencia y consumo, la relevancia no recae solamente en saber qué emisora lidera la cantidad de oyentes que posee. A esta variable conviene añadir el número de minutos de escucha para realizar un análisis fiel de la situación de la radiodifusión en España (Tabla 4). En este capítulo, la radio temática consigue, de nuevo, más minutos de escucha que la radio generalista, aunque la diferencias es de apenas ocho minutos al día.

Tabla 4. Minutos de escucha de la radio en España.

Fuente: Estudio General de Medios (EGM). Elaboración propia.

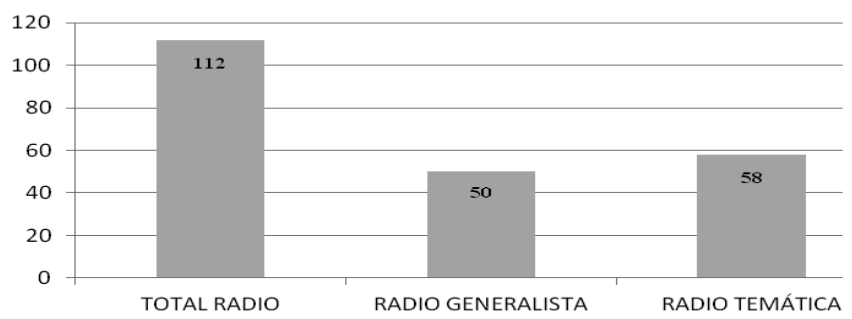

La Cadena Ser es la emisora generalista más escuchada de España, con 4.564.000 oyentes y un share del 35,50\% (Tabla 5). Le siguen Onda Cero, Cope y Radio Nacional. Si a estos datos añadimos las emisoras autonómicas, detectamos el éxito de Rac1 y Catalunya Ràdio en Cataluña (Tabla 6). Pese a que su ámbito de emisión -el de Catalunya Ràdio- se circunscribe a su territorio, una parte de la Comunidad Valenciana ${ }^{11}$ y Baleares, la penetración en el ámbito lingüístico catalán es evidente, lo que permite intuir una influencia política y de configuración de las agendas relevante.

${ }^{11}$ En el momento en que se realiza el trabajo de campo, Catalunya Ràdio todavía emitía en la Comunidad Valenciana. El pasado 21 de enero de 2014 la entidad Acció Cultural del País Valencià cerró sus repetidores cumpliendo el mandato del Ministerio de Industria. ENGUIX, SALVADOR. «Industria fuerza el apagón de Catalunya Ràdio en la Comunidad Valenciana.» La Vanguardia, 21 de 01 de 2014. 
Tabla 5. Oyentes de las cadenas de radio españolas (N) y su 'share' (\%).

Fuente: Estudio General de Medios (EGM). Elaboración propia.

\begin{tabular}{|l|r|r|}
\cline { 2 - 3 } \multicolumn{1}{c|}{} & \multicolumn{1}{c|}{$\begin{array}{c}\mathbf{N}^{\mathbf{0}} \\
\text { OYENTES }\end{array}$} & $\begin{array}{c}\text { SHARE } \\
(\%)\end{array}$ \\
\hline SER & 4.564 .000 & $35,50 \%$ \\
\hline ONDA CERO & 2.460 .000 & $19,50 \%$ \\
\hline COPE & 1.845 .000 & $13,10 \%$ \\
\hline RNE-1 & 1.235 .000 & $8,50 \%$ \\
\hline CATALUNYA RÀDIO & 646.000 & $3,50 \%$ \\
\hline EUSKADI IRRATIA & 61.000 & $0,60 \%$ \\
\hline RADIO EUSKADI & 146.000 & $1,20 \%$ \\
\hline RAC 1 & 744.000 & $5,50 \%$ \\
\hline CANAL SUR RADIO & 382.000 & $2,50 \%$ \\
\hline RADIO GALEGA & 180.000 & $1,20 \%$ \\
\hline
\end{tabular}

Tabla 6. Audiencia de las principales cadenas radiofónicas españolas.

Fuente: Estudio General de Medios (EGM). Elaboración propia

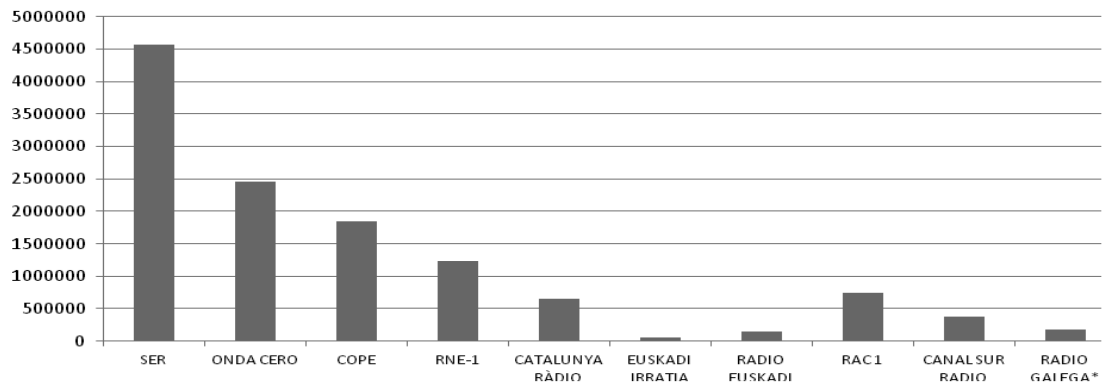

Algunos autores reclaman que las redes sociales intervengan en el proceso de medición de audiencia (Herrero Gutiérrez, 2011) para comprender mejor el comportamiento de un público cada vez más conectado a la emisión a la carta y también en línea "como complemento a las transmisiones hertzianas, como un servicio de valor añadido hacia los oyentes o como un medio para contactar con la audiencia y, así, obtener información o facilitar su participación" (Peñafiel Sainz, 2007). Para analizar cómo este éxito de audiencia convencional se traduce en participación e interacción social en Internet, este estudio de campo registró el índice Klout de los perfiles de Twitter de cada una de las cadenas el pasado 29 de noviembre de 2013, el día posterior a la publicación de la $3^{\mathrm{a}}$ Ola de 2013 del Estudio General de Medios. El índice Klout, como se ha visto anteriormente, mide a través de un algoritmo propio la influencia de los usuarios de Twitter en las redes sociales. En este proceso de "radiomorfosis" (Prata, 2008), la audiencia social de la radio jugará un papel cada vez más destacado con la aparición de "la radio social (por analogía con la 'televisión social') o la combinación del consumo radiofónico con la interacción en torno a éste en las redes sociales cuenta con un gran potencial"' (Videla Rodríguez y Piñeiro Otero, 2013). 
Tabla 7. Vínculos establecidos a través de la audiencia social en las principales cadenas españolas. Fuente: Klout. Elaboración propia.

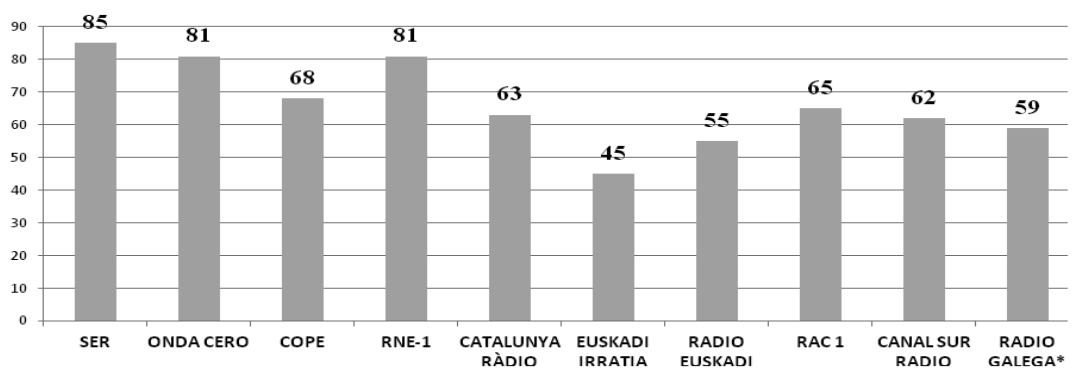

En términos de influencia en social media, la Cadena Ser es líder en España-igual que en número de oyentes- pese a que la diferencia respecto a sus competidores no es tan evidente como cuando analizamos los datos del Estudio General de Medios (Tabla 7). Así, parece que la generación de interacción no depende únicamente de la cantidad de personas que escuchan una cadena de radio sino de otras variables como pudieran ser la actividad de la audiencia en las redes sociales, la propia pro-actividad de las cadenas (Campos, 2008) y su estrategia social media, valores clave para la gestión de comunidades (Castelló Martínez, 2010). Este es un estudio descriptivo que simplemente pretende trazar la vinculación entre los datos de audiencia de las radios generalistas y su influencia 2.0 a través del índice Klout, lo que abre un campo de investigación al entorno de la configuración de los liderazgos en Internet a través de las interacciones con medios sociales y cómo se combina la capacidad de influencia en el entorno 2.0 y las emisiones hertzianas tradicionales.

Para contrastar lo que a simple vista parece una relación débil entre las variables de audiencia e índice Klout, es necesario estudiar los números absolutos y porcentajes de seguidores que tiene en Twitter cada cadena generalista, su índice de influencia y su audiencia según el EGM (Tabla 8).

Tabla 8. Cantidad de seguidores que tiene en Twitter cada cadena generalista, su índice de influencia y su audiencia según el EGM. Fuente: Klout y redes sociales.

Elaboración propia.

\begin{tabular}{|c|c|c|c|c|c|c|c|}
\hline CADENA & USUARIO & FOLLOWERS & $\%$ FOLLOWERS & KLOUT & $\begin{array}{c}\% \\
\text { KLOUT } \\
\end{array}$ & OYENTES & $\begin{array}{c}\% \\
\text { OYENTES } \\
\end{array}$ \\
\hline SER & @LL_SER & 333.857 & 46,0 & 85 & 12,8 & 4.564 .000 & 37,2 \\
\hline ONDA CERO & (a) OndaCero_es & 78.577 & 10,8 & 81 & 12,2 & 2.460 .000 & 20,1 \\
\hline COPE & (a) cope_es & 59.959 & 8,3 & 68 & 10,2 & 1.845 .000 & 15,0 \\
\hline RNE-1 & @)rne & 78.577 & 10,8 & 81 & 12,2 & 1.235 .000 & 10,1 \\
\hline CATALUNYA RÀDIO & (a)CatalunyaRadio & 58.389 & 8,0 & 63 & 9,5 & 646.000 & 5,3 \\
\hline EUSKADI IRRATIA & @euskadi_irratia & 6.623 & 0,9 & 45 & 6,8 & 61.000 & 0,5 \\
\hline RADIO EUSKADI & (a)radioeuskadi & 11.471 & 1,6 & 55 & 8,3 & 146.000 & 1,2 \\
\hline RAC 1 & (a)rac1 & 71.924 & 9,9 & 65 & 9,8 & 744.000 & 6,1 \\
\hline CANAL SUR RADIO & (a) canalsurradio1 & 4.237 & 0,6 & 62 & 9,3 & 382.000 & 3,1 \\
\hline RADIO GALEGA & (a)crtvg & 22.674 & 3,1 & 59 & 8,9 & 180.000 & 1,5 \\
\hline & & 726.288 & 100 & 664 & 100 & 12.263 .000 & 100 \\
\hline
\end{tabular}


La Cadena Ser es la que tiene más seguidores en la red de microblogging entre todas las radios generalistas españolas y acapara el $46 \%$ de followers, muy por encima de su inmediata competidora, Onda Cero. La influencia de ambas, en cambio, no es tan distante: la Cadena Ser dispone de un 85 en el índice Klout y Onda Cero y Radio Nacional la siguen con un 81 cada una, pese a sumar conjuntamente menos oyentes que la Ser. Para calcular la efectividad de su actividad en Twitter, dividiremos el número de seguidores por el índice Klout de cada cadena para saber cuántos seguidores corresponden a cada percentil de influencia (Tabla 9). La correlación de Pearson entre followers y Klout (pese a tratarse de una muestra muy reducida) es de 0.6164 , es decir, próxima a elevada (a partir de 0,7 ).

Tabla 9. Relación entre followers y Klout. Fuente: Klout y redes sociales. Elaboración propia.

\begin{tabular}{|l|r|r|r|}
\cline { 2 - 4 } \multicolumn{1}{c|}{} & FOLLOWERS & KLOUT & FOLL/KLOUT \\
\hline SER & 333.857 & 85 & $3.927,7$ \\
\hline ONDA CERO & 78.577 & 81 & 970,1 \\
\hline COPE & 59.959 & 68 & 881,8 \\
\hline RNE-1 & 78.577 & 81 & 970,1 \\
\hline
\end{tabular}

Traducida esta dinámica a la audiencia real en número de oyentes, comprobemos cuántos oyentes son necesarios para incrementar cada posición de Klout dividiendo el número de oyentes por el índice Klout (Tabla 10). Aquí la correlación es 0.5283, positiva pero menor que la anterior y no elevada.

Tabla 10. Relación entre oyentes y Klout. Fuente: Klout y redes sociales. Elaboración propia.

\begin{tabular}{|l|r|r|r|}
\cline { 2 - 4 } \multicolumn{1}{c|}{} & OYENTES & \multicolumn{1}{c|}{ KLOUT } & OYENT/KLOUT \\
\hline SER & 4.564 .000 & 85 & $53.694,1$ \\
\hline ONDA CERO & 2.460 .000 & 81 & $30.370,4$ \\
\hline COPE & 1.845 .000 & 68 & $27.132,4$ \\
\hline RNE-1 & 1.235 .000 & 81 & $15.246,9$ \\
\hline
\end{tabular}

Con estos datos, podemos comprobar que la Cadena Ser es la emisora que precisa de mayor número de seguidores para cada escalón que asciende en su índice de influencia. Pese a que es la emisora con mayor audiencia e influencia, es la menos eficiente en términos de influjo. Debemos apuntar una vez más, para contextualizar los datos, que el índice Klout obedece a un algoritmo que tiene en cuenta otros factores y que su fórmula permanece en secreto, aunque sea comúnmente aceptado como referencia. A este hecho cabe añadir que cada peldaño conseguido en el índice Klout es más difícil que el anterior en términos de interacción. En todo caso, centrándonos en followers y oyentes, observamos cifras de exigencia diferentes según el medio, lo que demuestra que otras variables ejercen influencia decisiva sobre el registro final. 


\section{Conclusiones}

El medio radiofónico continúa jugando un papel relevante en la población española que consume medios de comunicación. La radio generalista parece gozar de buena salud en lo que audiencia se refiere, aunque la temática musical va incrementando su penetración de manera significativa. La radio es un medio de implantación prácticamente paritaria, que resulta preeminente entre las capas superiores de los estratos socioeconómicos de la sociedad, con una penetración que supera el $70 \%$ en la clase alta mientras renueva sus oyentes con la incorporación de jóvenes que se vuelcan especialmente en la música.

La radio precisa de mecanismos de medición de su influencia en el entorno digital y aunque el índice Klout parece ser aceptado como indexador de los usuarios de Twitter, su algoritmo secreto arroja una clasificación que dista bastante del éxito que tiene cada cadena en términos de audiencia, medida a través del Estudio General de Medios. La interacción, el número de tweets y/o seguidores no es un reflejo fiel de la penetración de cada operador radiofónico, aunque arroje datos de interés para el estudio académico.

La emisora que mejor rentabiliza sus oyentes para transformarlos en influencia 2.0 es Radio Nacional de España-R1, que precisa únicamente de 15.246 oyentes por cada décima adquirida en el percentil Klout. La Cadena Ser lidera este barómetro, pero es enormemente ineficiente puesto que precisa de una enorme cantidad de seguidores y oyentes por cada nueva centésima del índice Klout. Pese a todo, su porcentaje de seguidores está por encima del porcentaje de oyentes que tiene. Onda Cero cuenta con un número de seguidores muy por debajo de lo que le correspondería de acuerdo con su audiencia, aunque su comunidad es activa y le proporciona buenos réditos de influencia. Radio Nacional es el operador más eficiente y que mayor rendimiento obtiene de su número de oyentes a la hora de trasladar esta cifra al total de seguidores y a su porcentaje de usuarios activos que mejoran su posición en el índice de influencia 2.0.

Tras el análisis comparado de estos datos, parece evidente que el indicador Klout, pese a ser importante en términos de comunicación 2.0, no se corresponde con el éxito de audiencia de cada emisora. Las correlaciones tienden a elevadas, pero no alcanzan esa condición. Su atribución y distribución apenas condiciona la audiencia real de las cadenas radiofónicas españolas, aunque sirva para monitorizar la influencia en redes sociales de su usuario de Twitter. Una emisora puede ser tremendamente activa e incluso conseguir un gran número de seguidores muy fieles sin que eso se traduzca necesariamente en una audiencia real comparable.

El uso de las redes sociales está cambiando las dinámicas de la participación de la audiencia (Herrera Damas, 2007) pero también de la transmisión de información. Las radios generalistas utilizan Twitter principalmente para transmitir noticias. A tenor de los datos analizados, parece evidente que debe hallarse un encaje de la audiencia social de la radio de igual forma que se empieza a hacer ya con la televisión como elemento adicional a las mediciones realizadas por el Estudio General de Medios, si bien es cierto que el éxito en términos de influencia digital no se traduce en una audiencia efectiva real.

Al tratarse de fenómenos nuevos en el entorno comunicativo y carecer de un registro evolutivo del índice Klout de cada emisora, conviene reivindicar la necesidad 
de mantener abierta esta línea de investigación para comprobar la evolución de estos indicadores y conjugarlos con otros que surgirán estableciendo una serie histórica que permita confrontar la relación entre followers, oyentes e influencia 2.0.

\section{Referencias bibliográficas}

ABEJÓN MENDOZA, Paloma; SASTRE ASENSIO, Ana; y LINARES RODRÍGUEZ, Virginia (2012): "Facebook y Twitter en campañas electorales en España". Anuario electrónico de Estudios en Comunicación Social Disertaciones, 5 (1).

AIMC (2013): Estudio General de Medios, Madrid, Asociación para la Investigación de los Medios de Comunicación.

ALEXANDER, Jenny (2013): "The case of the green vampire: eco-celebrity, twitter and youth engagement". Celebrity studies, 4(3), pp. 353-368.

ANGER, Isabel and KITTI, Christian (2011): "Measuring Influence on Twitter". IKnow, p. 31.

ARMSTRONG, Cory L. \& GAO, Fangfang (2010): "Now tweet this how news organizations use Twitter". Electronic News, 4 (4), pp. 218-235.

CAMPOS FREIRE, Francisco (2008): "Las redes sociales trastocan los modelos de los medios de comunicación tradicionales". Revista Latina de comunicación social, $11(63)$.

CASTELLÓ MARTÍNEZ, Araceli (2010): “Una nueva figura profesional: el Community Manager". Revista de la Red Académica Iberoamericana de Comunicación, Issue 1, pp. 74-97.

CASTELLS, Manuel (2001): La era de la información. Vol. I. La sociedad en red. México D.F., Siglo XXI.

CASTELLS, Pablo (2005): "La Web semántica. Sistemas Interactivos y Colaborativos en la Web". En: Sistemas interactivos y colaborativos en la web. Cuenca, Universidad de Castilla La Mancha.

CEBRIÁN HERREROS, Mariano (2008): La radio en internet. De la ciberradio a las redes sociales y la radio móvil. Buenos Aires, La Crujía.

CELAYA, Javier (2008): La empresa en la Wen 2.0. Barcelona, Gestión 2000.

CENTRE D'ESTUDIS D'OPINIÓ (2012): Baròmetre d'Opinió Política. Barcelona, CEO.

CHYI, Hsiang Iris \& CHADHA, Monica (2012): "News on new devices: Is miltiplatform news consuption a reality?". Journalism Practice, 6 (4), pp. 431-449.

COBB, Roger William \& ELDER, Charles D. (1972): Participation in American politics: the dynamics of agenda-building. s.l.:s.n.

COMAS, Eva (2006): Els recursos sonors en els informatius radiofònics (tesis doctoral). Barcelona, Universitat Ramon Llull.

COMSCORE (2013): Spain digital future in focus 2013. Madrid, ComScore. 
CONGOSTO, Mari Luz \& CLAES, Florencia (2013): “Análisis de la audiencia social por medio de Twitter. Caso de estudio: los premios Goya 2013”. Icono, 11 (14), pp. 53-82.

COYLE, Rebeca (2006): "Ether to 01 - Digitizing radio". Convergence: The International Journal of Research into New Media Technologies, Issue 12, pp. 123-127.

DURAN CASTELLS, Jaume \& SÁNCHEZ GOME, Lidia (2008): Industrias de la comunicación audiovisual. Barcelona, Universitat de Barcelona.

ELÓSEGUI, Tristán (2011): Los medios utilizan Twitter como altavoz, s.1.: s.n.

ENGUIX, Salvador (2014): "Industria fuerza el apagón de Catalunya Ràdio en la Comunidad Valenciana". La Vanguardia, 21 de enero.

FAUS BELAU, Ángel (2001): "La radio en el entorno cambiante del siglo XXI". En: Reinventar la radio. Pamplona, Eunate, pp. 15-37.

FERRERAS RODRÍGUEZ, Eva María (2011): "La estrategia de la corporación EITB (Euskal Irratia Telebisa) en Facebook y Twitter”. Razón y palabra, Issue 76.

FONDEVILA GASCÓN, Joan Francesc (2010): "Multimedia, digital ress and journalistic genres in Catalonia and in Spain: an empirical analysys". Communication Studies Journal, Issue 7, pp. 81-95.

FRANQUET, Rosa (2008): "El medio radiofónico". En: Industrias de la comunicación audiovisual. Barcelona, Universitat de Barcelona.

FRATICELLI, Damián (2011): “Las transformaciones de la programación de la radio cuando aparece la televisión: del oyente espectador al oyente radiofónico". $L a$ Trama de la Comunicación, Volumen 15.

GILLIN, Paul (2009): "The new influencers: a markeeter's guide to the social media". Linden.

GLEASON, Stephanie (2010): "Harnessing social media". American Journalism Review, 32 (1), pp. 6-7.

GONZÁLEZ CONDE, Ma Julia (2012): "La credibilidad de la voz como aspecto persuasivo de creación radiofónica". ICONO14. Revista científica de Comunicación y Tecnologías emergentes, 3 (2), pp. 140-169.

GONZÁLEZ MENDOZA, Vanessa \& PETERSEN, María Daniela (2010): "The reach of Twitter as a oplitical tool". Orbis: revista de ciencias humanas, Issue 6, p. 5.

GUTIÉRREZ ATALA, Fernando \& ARRIAGADA, Lely Zurita (2012): "Las redes sociales y su impacto en el proceso de preparación y producción de noticias: análisis de las rutinas de los periodistas a partir de tres diarios chilenos". Comunic@ación: Revista de Investigación en Comunicación y Desarrollo, Issue 3, pp. 36-44.

GUTIÉRREZ, María \& HUERTAS, Amparo (2003): "La programación de las radios generalistas en España". ZER, 8 (15), pp. 117-135.

HARBISON, Niall (2010): "10 ways journalists and the media use Twitter". The Next web. 
HERMIDA, Alfred (2010): "From TV to Twitter: How ambient news became ambient journalism". Media/Culture Journal, 13(2).

HERRERA DAMAS, Susana (2007): "La incidencia de Internet sobre la participación de los oyentes en los programas de radio". Redes.com, n ${ }^{\circ}$ 4, pp. 143-161.

HERRERA DAMAS, Susana \& REQUEJO, José Luis (2012): “Difundir información, principal uso que las emisoras de radio generalistas españolas están haciendo de Twitter". Observatorio, 6 (3), pp. 193-227.

HERRERA, Pau (2008): "Prosumer: Cuando el consumidor es el creativo". Revista $A P D$, Issue 236, pp. 77-79.

HERRERO GUTIÉRREZ, Francisco Javier (2011): "Los programas deportivos de la radio española en la red social Facebook: espacio de promoción, lugar de encuentro... ¿medidor de audiencia?”. Área Abierta, Issue 28, pp. 1-20.

HOLAHAN, Catherine (2008): "Facebook: no. 1 globally". Bussiness Week Online, Volumen 4.

HUGYES, Amanda Lee \& PALEN, Leysia (2009): “Twitter adoption and use un mass convergence and emergency events". International Journal of Emergency Management, 6 (3), pp. 248-260.

INSITES CONSULTING (2011): Social Media around the world. London, InSites.

JAVA, Akshay et al. (2007): Why we twitter: understanding microblogging usage and communities. Proceefimgs of the 9th Web KDD and 1st SNA-KDD 2007 workshop on Web mining and social network analysis. s.l.: AMC.

JEONG, Sun Ho (2013): Will Social Media save newspapers? Examining the effectiveness of Facebook and Twitter as news platforms. London, Routledge.

JU, Alice; JEONG, Sun Ho \& CHYI, Hsiang Iris (2013): "Will social media save newspapers. Examining the effectiveness of Facebook and Twitter as news platforms". Journalism Practice, pp. 1-17.

KANALLEY, Craig (2009): “The many ways journalists can use Twitter”. Twitterjournalism.

LIVINGSTON, Geoff (2010): "5 primary problems with Klout”. En: http://geofflivingston.com/2010/12/16/five-primary-problems-with-klout/

MARTÍNEZ COSTA, María del Pilar; MORENO, Elsa; y AMOEDO, Avelino (2012): "La radio generalista en la red: un nuevo modelo para la radio tradicional". Anagramas: Rumbos y sentidos de la comunicación, 10 (20), pp. 165-180.

MARWICK, Alice (2011): “To see and be seen: celebrity practice on Twitter". Convergence: The International Journal of Research into New Media Tecnologies, Issue 2, pp. 139-158.

MCCOMBS, Maxwell (2013): Setting the agenda: The mass media and public opinion. New York, John Wiley \& Sons. 
MEERMAN SCOTT, David (2010): The new rules of marketing and PR: how to use social media, blogs, news, releases, online video, and viral marketing to reach buyers. New Jersey, John Wiley \& Sons.

NAAMAN, Mor; BECKER, Hila \& GRAVANO, Luis (2011): "Hip and trendy: Characterizing emerging trends on Twiter". Journal of the American Society for Information Science and Technology, 62 (5), pp. 902-918.

NIETO, Margarita (1994): Sistemas de medición de la audiencia de radio en España. Barcelona, Universidad Autónoma de Barcelona.

O'CONOR, Rory (2012): Friends, followers and the future. How social media are changing politics, thereatening big brands, and killing traditional media. San Francisco, City Lights Books.

OLMSTEAD, Kenny; MITCHELL, Amy \& ROSENSTIEL, Tom (2011): "Navigating news online: where people go, how they get there and what lures them away". Pew Research Center's Project for Excellence in Journalism, Vol. 9.

ORIHUELA, José Luis (2011): Mundo Twitter. Barcelona, Alienta.

PARR, Ben (2009): “The New York Times hires a Social Media Editor, Does It need one?". Mashable, May, vol. 26.

PEÑAFIEL SAINZ, Carmen (Ed., 2007): Transformaciones de la radio y la televisión. Bilbao, Servicio de Publicaciones Universidad del País Vasco.

PERONA, José Juan (2009): "Edu-webs radiofónicas: experiencias españolas de educación en medios". Comunicar n ${ }^{\circ} 33$, v. XVII, pp. 107-114.

PICARD, Robert G. (2006): Journalism value creation and the future of news organizations. Cambridge, Joan Shorenstein Center om the Press, politics and Public Policy, John F. Kennedy School of GovernmentHarvard University.

POZZI, Sandro (2012): “Twitter se desnuda ante el mercado". El País, 4 octubre.

PRATA, Nair (2008): Webradio: novos géneros, novas formas de interacção. Belo Horizonte, Universidad Federal de Minas Gerais.

REQUEJO, José Luis \& HERRERA DAMAS, Susana (2011): ¿Cómo crear comunidad a través de Twitter? Nueve buenas prácticas en medios espalñoles, Bilbao, Universidad del País Vasco.

RODRÍGUEZ FIDALGO, Maribel \& SÁNCHEZ MERA, Antonio (2013): "La distribución de contenidos multipantalla, ¿ante nuevos contenidos mediáticos?: estudio de caso de RTVE. es en su móvil”. En: Notícias e Mobilidade: o jornalismo, na era dos dispositivos móveis. s.l.: Labcom-Laboratório de Comunicação e Conteúdos Online, pp. 265-292.

SCOLARI, Carlos Alberto (2009): "Transmedia storytelling: implicit consumers, narrative worlds, and branding in contemporany media production". International Journal of Communication, 3 (4), pp. 586-606.

SELLAS, Toni (2011): El Podcasting: la (r) evolución sonora. Barcelona, UOC. 
SELLAS, Toni (2013): "Radio y redes sociales: los magazines matinales en Twitter". Quaderns CAC, julio. XVI (39).

SERRANO PUCHE, Javier (2012): "Herramientas web para la mediación de la influencia digital: análisis de Klout y Peerindex". El profesional de la información, 21 (3), pp. 298-303.

STEPHENSON, Alan R.; REESE, David E. \& BEADLE, Mary E. (2013): Broadcast Announcing Worktext: A Media Performance Guide. Londres, Taylor \& Franci.

TORRES VILATARSANA, Marta (2004): "Com es regula la comunicació interpersinal a Internet? La 'netiqueta"'. Llengua i ús: revista tècnica de política lingüística, Issue 29, pp. 37-42.

TRAVER VALLÉS, Paula \& COBARSÍ MORALES, Josep (2012): “El uso de Twitter en los congresos profesionales de información y documentación: Estudio de caso". Revista general de información y documentación, Issue 22, pp. 349-365.

VIDELA RODRÍGUEZ, José Juan \& PIÑEIRO OTERO, Teresa (2013): "Hacia una radio social. interacción, proyección y repercusión de las cadenas españolas en las redes sociales". Icono 14, Volumen 11, pp. 83-113.

WATTS, Duncan (2004): “The New Science of Networks". Annual Review of Sociology, Issue 30, pp. 243-270.

ZARRELLA, Dan (2010): The social media marketing book. Sebastopol, O'Reilly.

ZHANG, Jinxue, et al. (2013): "On the impact of social botnets for Spain distribution and Digital influence Manipulation". Communications and Network Security (CNS). En: http://www2.hawaii.edu/ ruizhang/paper/zhang-CNS13.pdf 